\title{
New tetraphenylpyridinium-based luminogens with aggregation-induced emission characteristics
}

\author{
WANG ZongTan ${ }^{1}$, FANG Yuan $^{1}$, SUN JingZhi ${ }^{1}$, QIN AnJun ${ }^{1 *} \&$ TANG Ben Zhong ${ }^{1,2,3^{*}}$ \\ ${ }^{1}$ MOE Key Laboratory of Macromolecular Synthesis and Functionalization, Department of Polymer Science and Engineering, \\ Zhejiang University, Hangzhou 310027, China \\ ${ }^{2}$ Department of Chemistry, Institute for Advanced Study, Institute of Molecular Functional Materials, The Hong Kong University of \\ Science \& Technology (HKUST), Clear Water Bay, Kowloon, Hong Kong, China \\ ${ }^{3}$ Guangdong Innovative Research Team, SCUT-HKUST Joint Research Laboratory, State Key Laboratory of Luminescent Materials and \\ Devices, South China University of Technology (SCUT), Guangzhou 510640, China
}

Received June 26, 2013; accepted June 30, 2013; published online July 26, 2013

\begin{abstract}
The research on aggregation-induced emission (AIE) has drawn increasing interests in the past decade. With the efforts scientists paid, a variety of AIE systems have been developed, among which the tetraphenylethelene and silole derivatives are the most studied. Development of new AIE systems could further enrich the AIE molecules and promote the development of AIE area. In this communication, we prepared a new AIE system based on 1,2,4,6-tetraphenylpyridinium ions according to the restriction of intramolecular rotation mechanism. These molecules could be facilely synthesized via one-step and one-pot reaction. The ionic AIE-active molecules could find wide application in sensing and optoelectronic areas.
\end{abstract}

aggregation-induced emission, cation, restriction of intramolecular rotation, tetraphenylpyridinium

\section{Introduction}

Development of efficient luminescent materials in the solidstate is a hot research topic because of their promising practical applications in organic light-emitting diodes (OLEDs), organic solid-state lasers, chemical sensors, biological probes, logic circuitry, etc [1-6]. With the enthusiastic efforts scientists paid, tremendous fluorogenic dyes have been generated. However, many of them are highly emissive when dissolved in good solvents but faintly luminescent or totally nonemissive when increased the concentration or aggregated in poor solvents. This is the thorny problem of aggregation-caused quenching (ACQ) effect, which should be properly tackled because these materials are commonly

*Corresponding authors (email: qinaj@zju.edu.cn; tangbenz@ust.hk) used as solid films in their real-world applications [7, 8]. Various chemical, physical, and engineering approaches (e.g. attachment of alicyclic pendants, encapsulation by amphiphilic surfactants, and blending with transparent polymers) have been taken to alleviate the ACQ effect. These attempts, however, have met with limited success [9-11]. Dye aggregation is a spontaneously occurred physical phenomenon in nature. It will be better if the dye aggregation plays a positive instead of negative role for its emission in condensed phase. Thus, researchers never need to painfully fight against this process but amusedly utilize it to generate efficient emitters in the solid state [12-14].

We have observed an unusual phenomenon of "aggregation-induced emission" (AIE) in 2001 [15], which is exactly opposite to the aforementioned ACQ effect. A series of propeller-shaped molecules are nonluminescent when molecularly dissolved but become highly emissive in the aggregate state. Restriction of intramolecular rotation (RIR) of their multiple phenyl rings in the aggregate or solid states 
has been proven experimentally and theoretically as the main cause for the AIE effect [16-25].

Attracted by the intriguing photophysical process of AIE effect, the researchers throughout the world have been actively involved in this area. As a result, a variety of AIE systems have been developed in the past decade, among which the tetraphenylethelene (TPE) and silole derivatives have been widely investigated and identified as the archetype AIE systems [12, 13, 26]. They have been widely applied in fluorescent chemosensors and bioprobes, stimuliresponsive nanomaterials, and as active layers in efficient OLEDs [27]. However, the emission efficiency of TPEs and the tedious synthesis and purification procedures of siloles have greatly limited the expansion of the researches on AIE. To further promote the development of the AIE area, new AIE system is highly demanded. Furthermore, for biological applications, the AIE molecules have always been decorated with hydrophilic groups on their periphery to make them water soluble, which requires additional functionalization procedures. [28, 29].

We are interesting in exploring new AIE systems, especially the ionic AIE-active molecules because of their promising application in biological field. According to the RIR mechanism of AIE, a propeller-shaped molecule with multiple phenyl rotors could feature such characteristics, and pyridine derivatives could generate ionic species by substitution reaction with ease. Following these rules, a type of cationic molecules named 1,2,4,6-tetraphenylpyridinium drew our attention [30-32]. In such molecule, four phenyl rings are integrated on a pyridinium cation. We thus anticipated that the rotation of these phenyl rings in solution would consume the energy of excited state and make the molecule nonemissive or weakly luminescent. While, in the aggregate or solid states, the rotations are greatly restricted physically, and the emission will turn on.

\section{Results and discussion}

The 1,2,4,6-tetraphenylpyridinium-based ionic molecules could be facilely synthesized. As shown in Scheme 1, the reactions between 2,4,6-triphenylpyrylium tetrafluoroborate and mono- or di-amines in the refluxed ethanol solutions readily furnished corresponding compounds 1, 2 and $\mathbf{3}$ after simply workup procedures [33]. Unlike other ionic AIE-active luminogens, which were prepared via postfunctionalization reactions, the cationic pyridiniums are formed in situ with the substitution reactions of oxygen by nitrogen. The molecules are soluble in dichloromethane and other polar solvents like DMSO and DMF, but partially soluble in water. The molecules were fully characterized by ${ }^{1} \mathrm{H}$ (Figure S1) and ${ }^{13} \mathrm{C}$ (Figure S2) NMR and mass spectra (Figure S3), and satisfactory data corresponding their structures were obtained [see Supporting Information for details].

Then, we studied their photophysical properties. As can be seen from Figure S4, the dilute DMSO solutions of 1, 2 and 3 show absorption maximum $\left(\lambda_{\max }\right)$ at 309,313 , and $317 \mathrm{~nm}$, respectively. The red-shift of the $\lambda_{\max }$ is probably due to the conjugation extension from $\mathbf{1}$ to 3 .

According to the RIR mechanism of AIE, the 1,2,4,6tetraphenylpyridinium derivatives should possess such

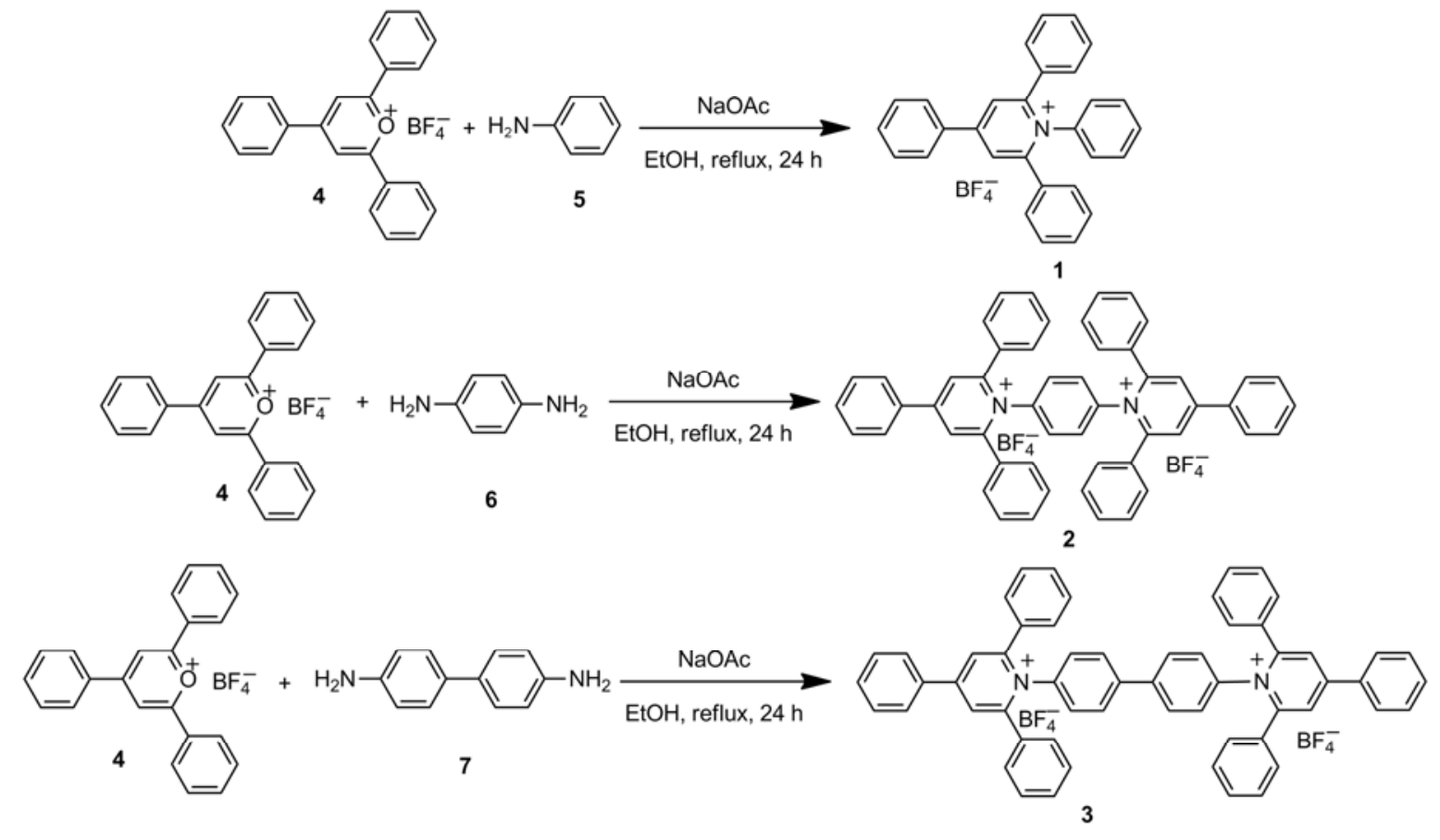

Scheme 1 Synthetic routes to 1,2,4,6-tetraphenylpyridiniums 1, 2 and $\mathbf{3}$. 

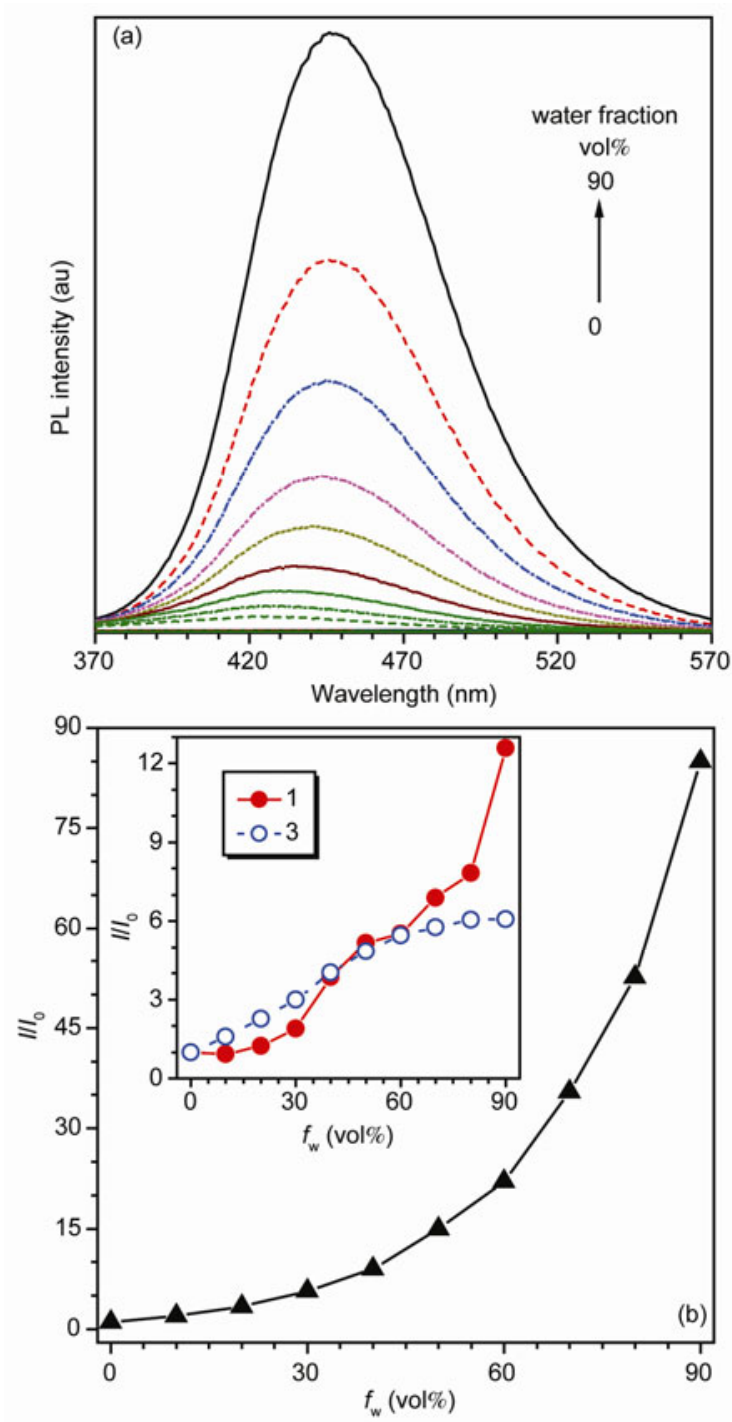

Figure 1 (a) PL spectra of 1,2,4,6-tetraphenylpyridinium derivative 2 in the DMSO/ $\mathrm{H}_{2} \mathrm{O}$ mixtures with different fractions of water $\left(f_{\mathrm{w}}\right)$. Concentration: $10 \mu \mathrm{M}$; excitation wavelength: $313 \mathrm{~nm}$. (b) Plot of the change in PL intensity with $f_{\mathrm{w}}$ of 1,2,4,6-tetraphenylpyridinium derivative 2 , where $I_{0}$ and $I$ are the maxima PL intensity. Inset: Plots of the changes in PL intensity with $f_{\mathrm{w}}$ of 1,2,4,6-tetraphenylpyridinium derivatives $\mathbf{1}$ and $\mathbf{3}$.

characteristics. We thus investigated the photoluminescence (PL) behaviors of $\mathbf{1}, \mathbf{2}$ and $\mathbf{3}$ in the solution and aggregate states. The aggregates were prepared by adding water into their DMSO solutions under vigorous stirring. The resultant mixtures are visually transparent and macroscopically homogenous, suggesting that the molecular aggregates are nanometer-sized [34].

Variations of the PL spectra of 2 with $f_{\mathrm{w}}$ in the DMSO/water mixtures are shown in panel (a) of Figure 1 as an example. When excited at $313 \mathrm{~nm}$, the PL spectrum of the diluted DMSO solution of $\mathbf{2}$ gives almost a flat line parallel to the abscissa, manifesting that $\mathbf{2}$ is a weak emitter when molecularly dissolved. In contrast, when a large amount of water is added into the solution, intense emission was recorded. Furthermore, the emission peaks were gradually red shifted from 422 to $446 \mathrm{~nm}$ under the same measurement conditions, which is probably due to the solvent polarity effect with more water added. Since $\mathbf{2}$ is partially soluble in water, addition of large amount water into its DMSO solution will make the molecules aggregated. 2 was thus induced to emit by aggregate formation, i.e. $\mathbf{2}$ is AIE-active. While, for $\mathbf{1}$ and $\mathbf{3}$, their DMSO solutions could emit weak light but the intensities were strengthened upon addition of water (Figures S5 and S6). In other words, they feature the characteristics of aggregate-enhanced emission (AEE).

The plots of relative emission intensity of the molecules in DMSO/water mixtures vs. $f_{\mathrm{w}}$ further confirm their AIE/AEE features. As shown in Figure 1(b), the emission intensity of 1, 2 and 3 was gradually increased with addition of water, and the highest values were all recorded in DMSO/water mixtures with $f_{\mathrm{w}}$ of $90 \%$, which are 12.6, 85.0, and 6.1-fold higher than that those in their DMSO solutions, respectively. The relatively low intensity enhancement of $\mathbf{1}$ and $\mathbf{3}$ is because their solutions are weakly emissive. These results indicate that the emission behaviors of 1,2,4,6-tetraphenylpyridinium-based molecules could be easily fine-tuned through molecular engineering.

\section{Conclusions}

In summary, according to the RIR mechanism, we have developed a new AIE system based on 1,2,4,6-tetraphenylpyridinium ion. Compounds $\mathbf{1}, \mathbf{2}$ and $\mathbf{3}$ could be facilely synthesized from commercially available starting materials of 2,4,6-triphenylpyrylium tetrafluoroborate and mono- or di-amines in the refluxed ethanol solutions. The photophysical measurements showed that these molecules possess AIE/AEE characteristics, which in turn proves the reasonability of the RIR mechanism. Unlike the reported ionic AIE-active molecules, in which the charges were on their periphery, these molecules have charges on their cores, which are promising to be applied as chemosensors and bioprobes with high sensitivity. The generation of more AIE-active 1,2,4,6-tetraphenylpyridinium ions and exploration of their applications are on-going in our research groups.

This work was partially supported by the National Science Foundation of China (21222402, 21174120, 20974098), the National Basic Research Program of China (2013CB834702, 2009CB623605), and the Research Grants Council of Hong Kong (603509, HKUST2/CRF/10, 604711, NHKUST620/11).

1 Shimizu M, Hiyama T. Organic fluorophores exhibiting highly efficient photoluminescence in the solid state. Chem Asian J, 2010, 5: 1516-1531

2 Liu B, Dan T, Bazan GC. Collective response from a cationic tetra- 
hedral fluorene for label-free DNA detection. Adv Funct Mater, 2007, 17: $2432-2438$

3 Chen Q, Wang JX, Yang F, Zhou D, Bian N, Zhang XJ, Yan CG, Han BH. Tetraphenylethylene-based fluorescent porous organic polymers: Preparation, gas sorption properties and photoluminescence properties. J Mater Chem, 2011, 21: 13554-13560

4 Chi ZG, Zhang XQ, Xu BJ, Zhou X, Ma CP, Zhang Y, Liu SW, Xu JR. Recent advances in organic mechanofluorochromic materials. Chem Soc Rev, 2012, 41: 3878-3896

5 Ning ZJ, Chen Z, Zhang Q, Yan YL, Qian SX, Cao Y, Tian H. Aggregation-induced emission (AIE)-active starburst triarylamine fluorophores as potential non-doped red emitters for organic light-emitting diodes and $\mathrm{Cl}_{2}$ gas chemodosimeter. Adv Funct Mater, 2007, 17: 3799-3807

6 Teng MJ, Jia XR, Yang S, Chen XF, Wei Y. Reversible tuning luminescent color and emission intensity: a dipeptide-based light-emitting material. Adv Mater, 2012, 24: 1255-1261

7 Lee SH, Jang BB, Kafafi ZH. Highly fluorescent solid-state asymmetric spirosilabifluorene derivatives. J Am Chem Soc, 2005, 127: 9071-9078

8 Aldred MP, Li C, Zhang GF, Gong WL, Li ADQ, Dai YF, Ma DG, Zhu MQ. Fluorescence quenching and enhancement of vitrifiable oligofluorenes end-capped with tetraphenylethene. J Mater Chem, 2012, 22: 7515-7528

9 Wang J, Zhao Y, Dou YC, Sun H, Xu P, Ye K, Zhang J, Jing S, Li F, Wang Y. Alkyl and dendron substituted quinacridones: Synthesis, structures, and luminescent properties. J Phys Chem B, 2007, 111: 5082-5089

10 Hecht S, Fréchet JMJ. Dendritic encapsulation of function: Applying nature's site isolation principle from biomimetics to materials science. Angew Chem Int Ed, 2001, 40: 74-91

11 Chen L, Xu S, McBranch D, Whitten D. Tuning the properties of conjugated polyelectrolytes through surfactant complexation. $J \mathrm{Am}$ Chem Soc, 2000, 122: 9302-9303

12 Hong YN, Lam JWY, Tang BZ. Aggregation-induced emission. Chem Soc Rev, 2011, 40: 5361-5388

13 Hong YN, Lam JWY, Tang BZ. Aggregation-induced emission: Phenomenon, mechanism and application. Chem Commun, 2009, 4332-4353

14 Qin AJ, Lam JWY, Tang BZ. Luminogenic polymers with aggregationinduced emission characteristics. Prog Polym Sci, 2012, 37: 182-209

15 Luo JD, Xie ZL, Lam JWY, Cheng L, Chen HY, Qiu CF, Kwok HS, Zhan XW, Liu YQ, Zhu DB, Tang BZ. Aggregation-induced emission of 1-methyl-1,2,3,4,5-pentaphenylsilole. Chem Commun, 2001, $1740-1741$

16 Chen JW, Law CCW, Lam JWY, Dong YP, Lo SMF, Williams ID, Zhu DB, Tang BZ. Synthesis, light emission, nanoaggregation, and restricted intramolecular rotation of 1,1-substituted 2,3,4,5tetraphenylsiloles. Chem Mater, 2003, 15:1535-1546

17 Zhang S, Qin AJ, Sun JZ, Tang BZ. Progress in mechanism study of aggregation-induced emission. Prog Chem, 2011, 23:623-636

18 Li Z, Dong YQ, Mi BX, Tang YH, Haussler M, Tong H, Dong YP, Lam JWY, Ren Y, Sung HHY, Wong KS, Gao P, Williams ID, Kwok HS, Tang BZ. Structural control of the photoluminescence of silole regioisomers and their utility as sensitive regiodiscriminating chemosensors and efficient electroluminescent materials. $J$ Phys Chem B, 2005, 109:10061-10069

19 Zeng Q, Li Z, Dong YQ, Di CA, Qin AJ, Hong YN, Ji L, Zhu ZC, Jim CKW, Yu G, Li QQ, Li ZA, Liu YQ, Qin JG, Tang BZ. Fluores cence enhancements of benzene-cored luminophores by restricted intramolecular rotations: AIE and AIEE effects. Chem Commun, 2007, 70-72

20 Shi JQ, Chang N, Li CH, Mei J, Deng CM, Luo XL, Liu ZP, Bo ZS, Dong YQ, Tang BZ. Locking the phenyl rings of tetraphenylethene step by step: understanding the mechanism of aggregation-induced emission. Chem Commun, 2012, 48: 10675-10677

21 Li Y, Zhou NC, Zhang W, Zhang F, Zhu J, Zhang ZB, Cheng ZP, Tu YF, Zhu XL. Light-driven and aggregation-induced emission from side-chain azoindazole polymers. J Polym Sci Part A: Polym Chem, 2011, 49: 4911-4920

22 Yao H, Ashiba K. Highly fluorescent organic nanoparticles of thiacyanine dye: A synergetic effect of intermolecular $\mathrm{H}$-aggregation and restricted intramolecular rotation. $R S C A d v, 2011,1: 834-838$

23 Chien WL, Yang CM, Chen TL, Li ST, Hong JL. Enhanced emission of a pyridine-based luminogen by hydrogen-bonding to organic and polymeric phenols. RSC Adv 2013, 3: 6930-6938

24 Wu QY, Deng CM, Peng Q, Niu YL, Shuai ZG. Quantum chemical insights into the aggregation induced emission phenomena: A QM/MM study for pyrazine derivatives. J Comput Chem, 2012, 33: 1862-1869

25 He JT, Xu B, Chen FP, Xia HJ, Li KP, Ye L, Tian WJ. Aggregation-induced emission in the crystals of 9,10-distyrylanthracene derivatives: The essential role of restricted intramolecular torsion. $J$ Phys Chem C, 2009, 113: 9892-9899

26 Qian LJ, Zhi JG, Tong B, Yang F, Zhao W, Dong YP. Organic compounds with aggregation-induced emission. Prog Chem, 2008, 20: 673-678

27 Zhao GS, Shi CX, Guo ZQ, Zhu WH, Zhu SQ. Recent application progress on aggregation-induced emission. Chin J Org Chem, 2012, 32: $1620-1632$

28 Wang M, Zhang GX, Zhang DQ, Zhu DB, Tang BZ. Fluorescent bio/chemosensors based on silole and tetraphenylethene luminogens with aggregation-induced emission feature. J Mater Chem, 2010, 20: 1858-1867

29 Ding D, Li K, Liu B, Tang BZ. Bioprobes based on AIE fluorogens. Acc Chem Res, 2013, 46: DOI: 10.1021/ar3003464

30 Miranda MA, Garcia H. 2,4,6-Triphenylpyrylium tetrafluoroborate as an electron-transfer photosensitizer. Chem Rev, 1994, 94, 1063-1089

31 Perez RT, Sanchez-Pedreno C, Ortuno JA. 1,2,4,6-Tetraphenylpyridinium perchlorate as a reagent for ion-association complex formation and its use for the spectrophotometric determination of thallium. Analyst, 1982, 107: 185-189

32 Han FL, Lu Y, Zhang Q, Sun JF, Zeng XS, Li CX. Homogeneous and sensitive DNA detection based on polyelectrolyte complexes of cationic conjugated poly(pyridinium salt)s and DNA. J Mater Chem, 2012, 22: 4106-4112

33 Fortage J, Tuyèras F, Ochsenbein P, Puntoriero F, Nastas F, Campagna S, Griveau S, Bedioui F, Ciofini I, Lainé PP. Expanded pyridiniums biscyclization of branched pyridiniums into their fused polycyclic and positively charged derivatives-assessing the impact of pericondensation on structural, electrochemical, electronic, and photophysical feature, Chem Eur J, 2010, 16: 11047-1063

34 Wang J, Mei J, Hu RR, Sun JZ, Qin AJ, Tang BZ. Click synthesis, aggregation-induced emission, E/Z isomerization, self-organization, and multiple chromisms of pure stereoisomers of a tetraphenylethene-cored luminogen. J Am Chem Soc, 2012, 134: 9956-9966 